\title{
Maturation of digestive function is retarded and plasma antioxidant capacity lowered in fully weaned low birth weight piglets
}

\author{
Joris Michiels ${ }^{1,2 *}$, Maartje De $\operatorname{Vos}^{3}$, Joris Missotten ${ }^{2}$, Anneke Ovyn ${ }^{2}$, Stefaan De Smet ${ }^{2}$ and \\ Christa Van Ginneken ${ }^{3}$ \\ ${ }^{1}$ Faculty of Biosciences and Landscape Architecture, University College Ghent, Valentin Vaerwyckweg 1, \\ 9000 Ghent, Belgium \\ ${ }^{2}$ Laboratory for Animal Nutrition and Animal Product Quality, Department of Animal Production, Ghent University, \\ Proefhoevestraat 10, 9090 Melle, Belgium \\ ${ }^{3}$ Laboratory for Veterinary Anatomy, Embryology and Pathology, Department of Veterinary Sciences, \\ University of Antwerp, Universiteitsplein 1, Wilrijk, Belgium
}

(Submitted 1 November 2011 - Final revision received 1 February 2012 - Accepted 1 February 2012 - First published online 3 April 2012)

\begin{abstract}
The digestive function of low birth weight (LBW) pigs post-weaning has been poorly studied. Therefore, newborns from eleven hyperprolific sows were weighed, weaned at $27 \cdot 2 \mathrm{~d}$ and fed a starter diet until sampling. Sampling was done between 18 and $28 \mathrm{~d}$ post-weaning. An LBW piglet ( $n$ 19) was defined as a piglet having a birth weight less than $1 \mathrm{~kg}$ and less than the lower quartile of litter birth weights. Normal birth weight (NBW) piglets ( $n$ 13) were having a birth weight close to the mean litter birth weight. For each piglet, eighty-eight variables were determined. Data were analysed with linear models with type of piglet and litter as predictors. A principal component analysis was performed to determine the most important discriminating variables. In the LBW pig, the development of the digestive tract postweaning was delayed: lower small-intestinal weight:length ratio due to a thinner tela submucosa and tunica muscularis and a higher secretory capacity, both in the distal jejunum. These observations might be a consequence of lower circulating insulin-like growth factor-1 (IGF-1) concentrations (126 (SE 10.0) v. 158 (SE 12.0) ng/ml for LBW and NBW, respectively) and a lower density of IGF-1 receptors in the proximal small intestine. Additionally, the plasma antioxidant capacity was lower for the LBW pig. Taken together, in the LBW piglet, the normal gut maturation post-weaning was retarded and this did not seem to be related to the weaning transition as such.
\end{abstract}

\section{Key words: Gastro-intestinal tract: Digestion: Intra-uterine growth restriction: Piglets}

Low birth weight (LBW) pigs, relative to normal birth weight (NBW) littermates, have lower birth weights and exhibit lower vitality scores $^{(1,2)}$. At birth, small pigs have less body energy reserves, take longer to achieve their first suckling, and are more prone to experience hypothermia and to be overlain by the sow. Within the litter, they are less able to access maternal resources than their heavier littermates. Overall, these factors are associated with a higher postnatal mortality and a lower growth rate in surviving LBW pigs ${ }^{(3-6)}$. LBW is a condition that stems from developmental and growth restriction during gestation $^{(7,8)}$. There is a body of evidence suggesting that compromised maternal-fetal relations are the primary cause of intra-uterine growth restriction (IUGR) ${ }^{(9,10)}$. To note, in the pig, IUGR occurs with a high frequency and its prevalence increases with increasing litter size ${ }^{(6)}$. In recent decades, selection for high litter size has resulted in a decrease in absolute mean birth weight and has generated a higher proportion of LBW piglets ${ }^{(2,6,11)}$. Prenatal interventions have been of limited success or were not cost-effective ${ }^{(8)}$. At present, most efforts are dedicated to adjust the nutritional management during the suckling and weaner phase to support survival and growth of these LBW pigs ${ }^{(8,12)}$

There is a plethora of peer-reviewed papers about LBW pigs dealing with the peri- and postnatal physiology ${ }^{(13,14)}$. These papers subscribe to the postulation that the pig is a good model for the IUGR and preterm human ${ }^{(15)}$. An additional set of papers about LBW pigs describes the effect of IUGR on carcass and meat quality at slaughter ${ }^{(3)}$. Surprisingly, pig studies

Abbreviations: $C$, crypt depth; EGFR, epidermal growth factor receptor; FRAP, ferric-reducing ability of plasma; GIT, gastro-intestinal tract; GSH-Px, glutathione-peroxidase; IGF-1, insulin-like growth factor-1; IGF-1R, insulin-like growth factor-1 receptor; Isc, short-circuit current; IUGR, intra-uterine growth restriction; J1, $3 \mathrm{~m}$ distal to pylorus; J2, $3 \mathrm{~m}$ proximal to the ileo-caecal valve; LBW, low birth weight; NBW, normal birth weight; PCA, principal component analysis; SI, small intestine; $V$, villus height.

*Corresponding author: J. Michiels, fax +32 9 2424279, email joris.michiels@hogent.be 
focusing on the post-weaning development of LBW piglets are scarce. Weaning is known to be a very stressful event to the animal and many structural and functional changes take place in the gut during the post-weaning period, in particular in the small intestine $(\mathrm{SI})^{(16)}$. Several studies demonstrated that LBW pigs when starting enteral nutrition, experience more digestive disorders due to gut immaturity ${ }^{(17,18)}$. Hence, it is interesting to know whether LBW pigs suffer from similar anomalies after weaning when the diet switches from sow milk to commonly used solid feeds based on plant proteins and lipids and rich in starch, and whether these alterations persist after the weaning transition. Results of such experiments - when focusing on relevant markers of digestive functioning - will aid to adjust feeding strategies for the LBW pig. Hence, the aim of the present study was to understand the developmental and prevailing differences of the digestive function and plasma oxidative status of fully weaned LBW $v$. NBW piglets at 2 weeks after weaning. The latter time frame was chosen because most reports indicate that the adaptations in response to weaning are then completed ${ }^{(16)}$.

\section{Materials and methods}

\section{Animals and experimental design}

The experiment was carried out according to the guidelines of the Ethics Committee of Ghent University (Belgium) for the humane care and use of animals in research. Animals originated from a commercial herd with 140 hyper-prolific sows and a 3-week batch-farrowing management (Waarschoot). Piglets (Hypor hybrid $\times$ Piétrain) were taken from two different batches of farrowing sows (batch I and batch II). Mean sow parity, number of piglets born alive and number of piglets weaned for the litters from which piglets were selected, were 5.4 (SE 1.12), 15.5 (SE 2.29) and 12.7 (SE 1.70), respectively.
At birth, all piglets alive were immediately ear-tagged, weighed and their sex determined. The time needed for first colostrum intake was recorded. Then, $24 \mathrm{~h}$ after parturition, the piglets were weighed again to estimate colostrum intake according to Devillers et al. $^{(19)}$ and rectal temperature was measured. A minimum number of piglets (only heavy piglets) were cross-fostered to litters that were not part of the study. From day 5 , piglets had free access to a commercial creep feed. At weaning $(27 \cdot 2$ (SE 1.27) d after parturition), piglets were weighed and transferred to the weaner unit whereby littermates stayed together. Then, piglets were fed a commercial diet composed of cereals, wheat gluten, toasted soyabeans, soyabean meal, soyabean oil, whey, lactose and nutritional additives such as amino acids, minerals and vitamins (analysed proximate analysis: DM, $889 \mathrm{~g} / \mathrm{kg}$; crude protein, $182 \mathrm{~g} / \mathrm{kg}$; ether extract, $57 \mathrm{~g} / \mathrm{kg}$ and crude fibre, $68 \mathrm{~g} / \mathrm{kg}$ and calculated metabolisable energy $15.6 \mathrm{MJ} / \mathrm{kg}$ ) ad libitum until the day of sampling. The sampling day was chosen at least 2 weeks post-weaning (varying between 18 and $28 \mathrm{~d}$ post-weaning) because many reports related to pigs highlight that most of the functional and structural changes of the gut in response to weaning are completed within 2 weeks ${ }^{(16)}$. Then, pairs of LBW and NBW littermates were selected and transferred to the laboratory for sampling. Selection criteria were based on birth weight and weight at the time of sampling (Table 1). An LBW piglet was defined as a piglet having a birth weight less than $1 \mathrm{~kg}$ and less than the lower quartile of litter birth weights. Nearly $21 \%$ of the piglets in the litters matched this criterion at the time of birth. Furthermore, LBW pigs had a longer time for first colostrum intake (lower vitality at birth), a lower colostrum intake and a lower $24 \mathrm{~h}$ rectal temperature. The pre-weaning mortality within this group of piglets was $34 \cdot 3 \%$. NBW littermates were having a birth weight close to the mean litter birth weight. In addition, at sampling, LBW and NBW piglets needed to

Table 1. Observations in the suckling and weaner phase of low birth weight (LBW) and normal birth weight (NBW) piglets

(Mean values with their standard errors)

\begin{tabular}{|c|c|c|c|c|c|}
\hline \multirow[b]{2}{*}{ Variable } & \multicolumn{2}{|c|}{ LBW } & \multicolumn{2}{|c|}{ NBW } & \multirow[b]{2}{*}{$P$} \\
\hline & Mean & SE & Mean & SE & \\
\hline Time to suck first colostrum (min) & 50 & $5 \cdot 0$ & 34 & 5.5 & 0.041 \\
\hline Estimated colostrum intake* $(\mathrm{ml})$ & 349 & $13 \cdot 8$ & 452 & $15 \cdot 5$ & $<0.001$ \\
\hline $24 \mathrm{~h}$-rectal temperature $\left({ }^{\circ} \mathrm{C}\right)$ & $38 \cdot 7$ & 0.12 & $39 \cdot 1$ & 0.14 & 0.024 \\
\hline \multicolumn{6}{|l|}{ Body weight (g) } \\
\hline At birth & 912 & $21 \cdot 1$ & 1287 & $24 \cdot 1$ & $<0.001$ \\
\hline At weaning & 5610 & 140 & 7100 & 160 & $<0.001$ \\
\hline At sampling & 8150 & 180 & 10660 & 205 & $<0.001$ \\
\hline \multicolumn{6}{|l|}{ Average daily gain $(\mathrm{g} / \mathrm{d})$} \\
\hline Suckling phase & 179 & $5 \cdot 1$ & 222 & $5 \cdot 8$ & $<0.001$ \\
\hline Post-weaning phase & 119 & $7 \cdot 1$ & 168 & $8 \cdot 1$ & $<0.001$ \\
\hline \multicolumn{6}{|l|}{ Relative growth rate $(\mathrm{kg} / \mathrm{kg})$} \\
\hline Suckling phase & $5 \cdot 1$ & 0.16 & 4.5 & 0.19 & 0.030 \\
\hline Post-weaning phase & 0.47 & 0.030 & 0.52 & 0.034 & 0.277 \\
\hline \multicolumn{6}{|l|}{ Mortality $\ddagger(\%)$} \\
\hline Suckling phase & \multicolumn{2}{|c|}{$34 \cdot 3$} & \multicolumn{2}{|c|}{ ND } & \\
\hline
\end{tabular}

ND, not determined.

${ }^{\star}$ According to formula reported by Devillers et al. ${ }^{(19)}$.

† Relative growth rate; body weight gain/initial body weight.

$\ddagger$ Mortality of all piglets defined as LBW based on the birth weight criterion out of the litters used. 
have a relatively low and normal weight, respectively, in order to avoid the selection of piglets showing compensatory growth or runting animals. This second criterion was established because: (1) runting has been associated with an increased disease incidence and (2) growth compensation might be a result of changes in the intermediate metabolism. Our objective was to study alterations in the digestive function and hence we tried to avoid other factors affecting profoundly the animal's physiology. Yet, the number of animals that was excluded for this criterion in the present study was very limited. In total, nineteen LBW and thirteen NBW piglets were selected from eleven litters.

\section{Sampling}

Selected piglets were electrically stunned and euthanised by exsanguination. Blood was collected in EDTA (as anti-coagulant) tubes for the determination of the oxidative status in plasma and in plain tubes for insulin-like growth factor-1 (IGF-1) determination in serum. Afterwards, the gastro-intestinal tract (GIT), including the liver was removed. A segment of $20 \mathrm{~cm}$ of the SI, $3 \mathrm{~m}$ proximal to the ileo-caecal valve (J2), was taken and immediately processed for the Ussing chamber measurements. Subsequently, the GIT was partitioned into the following digesta sampling sites: stomach, proximal SI ( $\mathrm{S} 1,0-3 \mathrm{~m}$ distal from pylorus), distal SI (S2, 3-0 m proximal to ileo-caecal valve) and caecum. The content of each segment was quantitatively collected and the $\mathrm{pH}$ was determined. Aliquots were taken and acidified to $\mathrm{pH}<2$ with $2 \%(\mathrm{w} / \mathrm{w})$ of $6 \mathrm{M}-\mathrm{H}_{2} \mathrm{SO}_{4}$ to stop fermentation and stored at $-20^{\circ} \mathrm{C}$ pending analysis for bacterial metabolites. The empty weight of digestive organs and length of SI were measured. From proximal ( $3 \mathrm{~m}$ distal to pylorus; J1) and distal (3 m proximal to the ileo-caecal valve; J2) SI, multiple segments $(10 \mathrm{~cm}$ of length each) were taken and flushed with $0.9 \%(\mathrm{w} / \mathrm{v})$ saline and either snap-frozen in liquid $\mathrm{N}_{2}$ and stored at $-80^{\circ} \mathrm{C}$ or fixed in neutral buffered formalin pending analysis.

\section{Determination of variables}

For each piglet, eighty-eight variables were determined including size of digestive organs (twelve variables; for definitions of organs and parts of organs, see previous section), SI histomorphology (12), digesta characteristics (23), distal SI secretory and absorptive capacity (4), distal SI barrier function (2), SI brush-border enzyme activities (12), SI growth-factor receptors (10), SI cytokine concentrations (8), plasma oxidative status (4) and serum IGF-1 (1).

Measurements of the histo-morphological parameters, villus height $(V)$ and crypt depth $(C)$, for the formalin-fixed samples of $\mathrm{J} 1$ and $\mathrm{J} 2$ were carried out as described by Van Nevel et $a l .{ }^{(20)}$. Briefly, after dehydration and impregnation with paraffin, $4 \mu \mathrm{m}$ sections (six per piglet and intestinal site) were stained with haematoxylin and eosin. $V$ (from tip to base) and $C$ (from base to opening) of well-oriented villi and adjacent crypts were measured using a microscope equipped with a camera and computer with appropriate software (Olympus, CX41/U30, software CellB). At least twenty $V-\mathrm{C}$ were measured per piglet and intestinal site. In addition, thickness of the tela submucosa and tunica muscularis was measured.

Bacterial metabolites were determined in the digesta of S2 and caecum. SCFA and lactic acid were analysed by a GC method described by Jensen et al. ${ }^{(21)}$ and modified by Missotten et al. ${ }^{(22)}$.

The distal SI secretory and absorptive capacity and barrier function were determined in Ussing chambers according to Michiels et al. ${ }^{(23)}$. Basal values (tissue resistance ( $\mathrm{Rt}_{\mathrm{BASAL}}$, $\Omega \mathrm{cm}^{2}$ ); basal short-circuit current (Isc $\mathrm{BASAL}, \mu \mathrm{A} / \mathrm{cm}^{2}$ )) were obtained in two chambers as average values between 15 and $20 \mathrm{~min}$. In addition, the changes in Isc $\left(\Delta \mathrm{Isc} ; \mu \mathrm{A} / \mathrm{cm}^{2}\right)$ due to the addition of $\mathrm{D}$-glucose and serotonin and theophylline as secretagogues were determined. Besides, two other chambers were used to measure permeability to the macro-molecular marker fluorescein isothiocyanate dextran (FD4, $1.0 \mathrm{mg} / \mathrm{ml}$; $4000 \mathrm{Da}$; Sigma-Aldrich) added at the mucosal side. Permeability was monitored by sampling the serosal compartment at $20 \mathrm{~min}$ intervals for $80 \mathrm{~min}$. The apparent permeation coefficient $\left(P_{\mathrm{app}}\right)$ was calculated as: $P_{\mathrm{app}}(\mathrm{cm} / \mathrm{s})=(\mathrm{d} c / \mathrm{d} t) \times V / c_{0} / A$, whereby $\mathrm{d} c / \mathrm{d} t$ is the change of serosal concentration in the 20- to $100-\mathrm{min}$ period (M/s); $V$ is the volume of the chamber, $c_{0}$ the initial marker concentration in the mucosal reservoirs and $A$ the area of the exposed intestine in the chambers $\left(\mathrm{cm}^{2}\right)$. Data on $P_{\text {app }}$ were $\log _{10}$ transformed before data analysis.

Frozen samples $\left(-80^{\circ} \mathrm{C}\right)$ of SI were homogenised and enzymatic activities were measured using a Tecan sunrise microtitre plate reader (Tecan Trading AG). Aminopeptidase A (EC 3.4.11.1), aminopeptidase N (EC 3.4.11.2) and dipeptidylpeptidase IV (EC 3.4.14.5) were kinetically measured by spectrophotometry using Glu-p-nitroaniline, L-alanine 4-nitro-anilide hydrochloride and H-glycyl-prolyl-p-nitroaniline p-tosylate as substrates, respectively. Lactase (EC 3.2.1.23), sucrase ( $E C$ 3.2.1.48) and maltase (EC 3.4.11.2) activities were determined with lactose $(120 \mathrm{~mm})$, sucrose $(10 \mathrm{~mm})$ and maltose $(11.2 \mathrm{~mm})$ as substrates. Enzyme activity levels were expressed as units per g wet tissue. Here, one unit of activity was defined as the amount of enzyme that hydrolyses $1 \mu \mathrm{mol}$ substrate per min.

Immunohistochemical staining was used to detect the presence and distribution of the epidermal growth factor receptor (EGFR) and the IGF-1 receptor (IGF-1R). All staining procedures were carried out at room temperature, unless otherwise stated. Paraffin sections $(5 \mu \mathrm{m})$ were hydrated and non-specific staining was blocked by incubating in $0.3 \%(\mathrm{w} / \mathrm{v}) \mathrm{H}_{2} \mathrm{O}_{2}$ in methanol for $30 \mathrm{~min}(\mathrm{EGFR})$ or in $1 \%(\mathrm{w} / \mathrm{v}) \mathrm{H}_{2} \mathrm{O}_{2}$ in methanol for $30 \mathrm{~min}$ (IGF-1R). In the case of EGFR-immunohistochemistry, sections were incubated with $1 \%(\mathrm{w} / \mathrm{v})$ sodium-borohydride for $5 \mathrm{~min}$, rinsed in $50 \mathrm{~mm}$-Tris (hydroxymethyl) aminomethane-buffered saline solution $(\mathrm{pH} 7 \cdot 4)$ with $1 \%(\mathrm{w} / \mathrm{v})$ bovine serum albumin (BSA) (Tris-buffered saline (TBS)/BSA) and subjected to a biotin blocking system (Dakocytomation) before incubating with the primary antibody. Sections were incubated with mouse monoclonal anti-EGFR (1/70 diluted in TBS/BSA) (Sigma) or rabbit polyclonal IGF-1R anti-serum (Santacruz Biotechnology) (1/100 diluted in PBS) overnight at $4^{\circ} \mathrm{C}$. Following rinsing in TBS/BSA (EGFR) or PBS (IGF-1R), the sections were incubated with biotinylated goat anti-mouse (1/400) (Dakocytomation) diluted in TBS/BSA for $60 \mathrm{~min}$ 
(EGFR) or were incubated with Envision anti-rabbit (Dakocytomation) for $30 \mathrm{~min}$ (IGF-1R) at room temperature. In order to visualise the presence of EGFR, a third step, namely incubating the sections after rinsing in TBS/BSA with streptavidin-conjugated horseradish peroxidase $(1 / 400)$ diluted in TBS for $30 \mathrm{~min}$ needed to be applied. In order to visualise both the EGFR and IGF-1R immunocomplexes, aminoethylcarbazol (Dakocytomation) was applied to the sections after rinsing the sections in TBS (EGFR) or PBS (IGF-1R) and aqua dest. Staining was semiquantitatively (intensity and extent of staining) scored ( 0 as no staining, 1 as weak staining, 2 as moderate staining, 3 as strong staining). EGFR was determined in the villi and goblet cells and IGF-1R in the muscularis, submucosal arterioles and enterocytes of proximal and distal SI tissues.

To determine the cytokine level in the small-intestinal tissue homogenates (samples stored at $-80^{\circ} \mathrm{C}$ ), commercial ELISA kits were used: a swine ELISA kit (KSC0102, Invitrogen) for IL-10 and porcine DuoSet ELISA Development systems for IL-1 $\beta$ (DY681, R\&D Systems Europe Limited), IL-6 (DY686, R\&D Systems Europe Limited) and TNF- $\alpha$ (DY690B, R\&D Systems Europe Limited). The tests were run according to the manufacturer's protocol. Results are expressed as $\mathrm{pg} / \mathrm{g}$ protein.

Overall, four parameters of oxidative status in plasma were determined. The ferric-reducing ability of plasma (FRAP) assay is considered as a measure of the 'total antioxidant power', according to the method described by Benzie \& Strain $^{(24)}$. The test is based on the reduction of the ferrictripyridyltriazine complex to the ferrous form at low $\mathrm{pH}$. The malondialdehyde concentration in plasma was measured by the thiobarbituric acid-reactive substances (TBARS) method, as described by Grotto et al. ${ }^{(25)}$. Assessment of glutathioneperoxidase (GSH-Px) activity follows the method described by Hernández et $a l .{ }^{(26)}$ and the guidelines of the catalogue of ZeptoMtrix Corporation (Total glutathione-peroxidase assay, description and intended use). $\alpha$-Tocopherol content in plasma was determined using HPLC according to Desai ${ }^{(27)}$.

Serum IGF-1 concentrations were determined using a commercial enzyme immune assay (Mediagnost, Inc.) following the manufacturer's protocol.

\section{Statistical analysis}

The effect of type of piglet (LBW $v$. NBW) was analysed using the generalised linear models procedure in IBM SPSS Statistics version 19.0 program for Windows (SPSS, Inc.). Continuous variables were linked in a linear way with the predictor variables and the categorical variables were linked through the multinomial logistic function. The predictor variables included type of piglet (LBW $v$. NBW) and litter to account for litter effects (all piglets born within a litter remained pen-mates until sampling). The interaction term was not included in the model since in nearly all cases the interaction term was not significant $(P>0.05)$. The number of LBW and NBW piglets per litter varied from one to four and one to two, respectively, which means that the final data set represents an unbalanced design (in total nineteen LBW and thirteen NBW piglets). Therefore, to calculate the sum of squares, type III was used. Data are presented as estimated marginal means with their standard errors.
Principal component analysis (PCA) was carried out in order to deduce the variables that contributed most to differences between LBW and NBW piglets. Therefore, the methodology as outlined by Montagne et $a l^{(16)}$ was applied. Briefly, the data of continuous variables (78) were standardised, i.e. the data were diminished by the mean and divided by the standard deviation of that variable. A first PCA, including a scree plot, was done to determine the number of principal components to be retained. Following this, seven principal components appeared before a clear break where the eigenvalues appeared to level off to the right of the plot. The eigenvalues of these seven principal components were greater than 3.0. Then, variables that did not load on any principal component retained (correlation coefficient between variable and principal components $\leq 0.5)$ were excluded. In addition, from those variables within a family of variables, which were highly correlated ( $r>0.55 ; P<0.05$ ), only the main representative variable (highest principal component loading) was retained for the final analysis. Overall, thirty-five variables were kept for this final PCA. All calculations were carried out using the IBM SPSS Statistics version 19.0 program for Windows (SPSS, Inc.).

\section{Results}

Differences of the digestive function and plasma oxidative status of fully weaned low birth weight v. normal birth weight piglets

Upon sampling, all piglets were without signs of respiratory disease or gastro-intestinal disorders. However, multiple LBW piglets were small for age and demonstrated poor growth. Absolute weight of all digestive organs was significantly lower for LBW piglets compared to NBW littermates with the exception of spleen, in contrast to the relative weight of these organs (Table 2). SI weight/total length was reduced in the LBW pig $(P=0.002)$. SI histo-morphological variables were not affected by birth weight, except for the thickness of tela submucosa and tunica muscularis in the distal SI (Table 3). Both parameters were significantly reduced by approximately $16-18 \%$ in the LBW pigs. Neither absolute nor relative fresh matter content of the gut compartments was affected by the type of piglet (Table 4). In the caecum, the fermentation pattern was clearly altered in the LBW pigs; lower $\mathrm{pH}$ (5.55 (SE 0.053) v. 5.74 (SE 0.056) for LBW and NBW pigs, respectively; $P=0.023$ ) concomitant with a higher SCFA concentration (17\%, $P=0.013)$ due to an increase in acetate and propionate production. The proportion of acetate as fermentation product in the caecum was increased in LBW pigs. Only one variable describing the barrier and secretory function of the distal SI was found to be different (data not shown). Isc $\mathrm{BASAL}_{\mathrm{BAL}}$ was markedly higher in LBW pigs $\left(-1.5\left(\operatorname{se~2.41)} v .-12.2(\operatorname{SE} 2.62) \mu \mathrm{A} / \mathrm{cm}^{2}\right.\right.$ for LBW and NBW pigs, respectively; $P=0.008)$. Neither carbohydrase nor peptidase brush-border enzyme activities were affected by birth weight. Scores on staining intensity of EGFR were not different among groups. With the exception of enterocytes in the distal SI, the presence of IGF-1R in intestinal tissues was substantially lower in the LBW animals, which reached significance in the proximal tissues. The scores for the muscularis 
Table 2. Size of digestive organs of fully weaned low birth weight (LBW) and normal birth weight (NBW) piglets (Mean values with their standard errors)

\begin{tabular}{|c|c|c|c|c|c|}
\hline \multirow[b]{2}{*}{ Variable } & \multicolumn{2}{|c|}{ LBW } & \multicolumn{2}{|c|}{ NBW } & \multirow[b]{2}{*}{$P$} \\
\hline & Mean & SE & Mean & SE & \\
\hline \multicolumn{6}{|l|}{ Stomach } \\
\hline Weight (g) & 85 & $3 \cdot 3$ & 105 & 3.4 & $<0.001$ \\
\hline Relative weight ( $\mathrm{g} / \mathrm{kg} \mathrm{BW}$ ) & 9.6 & 0.26 & $9 \cdot 2$ & 0.27 & 0.318 \\
\hline \multicolumn{6}{|l|}{ Small intestine } \\
\hline Total length (m) & 9.5 & 0.19 & $10 \cdot 3$ & 0.21 & 0.007 \\
\hline Weight (g) & 376 & 14.5 & 469 & $16 \cdot 6$ & $<0.001$ \\
\hline Relative weight ( $\mathrm{g} / \mathrm{kg} \mathrm{BW}$ ) & $42 \cdot 5$ & $1 \cdot 20$ & $41 \cdot 3$ & 1.37 & 0.502 \\
\hline Weight/total length $(\mathrm{g} / \mathrm{m})$ & 39.4 & 1.14 & $45 \cdot 6$ & 1.31 & 0.002 \\
\hline \multicolumn{6}{|l|}{ Caecum } \\
\hline Weight (g) & 24 & $1 \cdot 3$ & 32 & 1.5 & 0.001 \\
\hline Relative weight ( $/ \mathrm{kg} \mathrm{BW}$ ) & 2.7 & 0.12 & $2 \cdot 8$ & 0.14 & 0.521 \\
\hline \multicolumn{6}{|l|}{ Liver } \\
\hline Weight (g) & 252 & $8 \cdot 2$ & 305 & 9.4 & $<0.001$ \\
\hline Relative weight (g/kg BW) & $28 \cdot 2$ & 0.54 & $26 \cdot 8$ & 0.62 & 0.072 \\
\hline \multicolumn{6}{|l|}{ Spleen } \\
\hline Weight (g) & 21 & 1.0 & 24 & $1 \cdot 1$ & 0.065 \\
\hline Relative weight ( $/ \mathrm{kg} \mathrm{BW}$ ) & $2 \cdot 3$ & 0.11 & $2 \cdot 1$ & 0.11 & 0.113 \\
\hline
\end{tabular}

BW, body weight.

were 0.95 and $1.42(P=0.021)$, for the submucosal arterioles 0.53 and $0.97(P=0.023)$ and for the enterocytes 0.26 and 0.66 $(P=0.032)$ for the proximal SI tissues of LBW and NBW piglets, respectively. In addition to this, IGF-1 concentrations were 126 (se 10.0) v. 158 ( $\operatorname{se~12\cdot 0)~ng/ml~(~} P=0.034)$ for LBW and NBW pigs, respectively. The highest cytokine concentrations were observed for IL-1 $\beta$ (86 (SE 12.4) and 145 (SE 9.6) pg/g protein for proximal and distal SI, respectively); followed by IL-6 (122 (sE 14.4) and 78 (SE 11.8) pg/g protein), TNF- $\alpha$ (23 (SE 3.4) and $24(\operatorname{SE~} 1.7) \mathrm{pg} / \mathrm{g}$ protein) and IL-10 (1.0 (SE 0.27) and 2.3 ( $\operatorname{se} 0.24) \mathrm{pg} / \mathrm{g}$ protein). However, for none of these parameters birth weight affected the outcome. Finally, Table 5 represents parameters of the plasma oxidative status of fully weaned LBW $v$. NBW piglets. FRAP values $(-7 \%, P=0.049)$ and GSH-Px enzyme activity $(-13 \%, P<0.001)$ were reduced in LBW pigs.

\section{Principal component analysis}

Variables that did not load on any principal component retained were relative weight of the stomach, caecum and liver, relative weight of gastric and caecal contents, $\mathrm{pH}$ of gastric and proximal SI digesta, caecum propionate/SCFA, caecum butyrate/SCFA, caecum lactate, distal SI Papp ${ }_{\mathrm{FD} 4}$, proximal and distal SI lactase, distal SI IL-1 $\beta$, proximal SI IL-10 and plasma $\alpha$-tocopherol. Within the group of anatomical variables, several variables were highly correlated. For example, liver weight was correlated with stomach weight $(r 0 \cdot 81 ; P<0 \cdot 001)$, SI weight ( $r 0 \cdot 88 ; P<0 \cdot 001)$, caecum weight $(r 0.67 ; P<0.001)$, SI total length $(r 0.65 ; P<0.001)$, SI weight/total length $(r 0.79$; $P<0.001)$ and spleen weight $(r 0.63 ; P<0.001)$. From this group, only four variables entered the final PCA. Proximal and distal $V+\mathrm{C}$ and $V / C$ showed significant correlations with

Table 3. Small-intestinal histo-morphology of fully weaned low birth weight (LBW) and normal birth weight (NBW) piglets

(Mean values with their standard errors)

\begin{tabular}{|c|c|c|c|c|c|}
\hline \multirow[b]{2}{*}{ Variable } & \multicolumn{2}{|c|}{ LBW } & \multicolumn{2}{|c|}{ NBW } & \multirow[b]{2}{*}{$P$} \\
\hline & Mean & SE & Mean & SE & \\
\hline \multicolumn{6}{|l|}{ Proximal } \\
\hline$V(\mu \mathrm{m})$ & 497 & $14 \cdot 4$ & 504 & $16 \cdot 4$ & 0.732 \\
\hline$C(\mu \mathrm{m})$ & 341 & 8.0 & 338 & $9 \cdot 1$ & 0.781 \\
\hline$V+C(\mu \mathrm{m})$ & 838 & $20 \cdot 3$ & 842 & $23 \cdot 2$ & 0.896 \\
\hline$V / C$ & 1.49 & 0.034 & 1.53 & 0.039 & 0.412 \\
\hline Tela submucosa $(\mu \mathrm{m})$ & 44 & 1.6 & 47 & 1.8 & 0.287 \\
\hline Tunica muscularis $(\mu \mathrm{m})$ & 221 & 6.4 & 225 & $7 \cdot 3$ & 0.692 \\
\hline \multicolumn{6}{|l|}{ Distal } \\
\hline$V(\mu \mathrm{m})$ & 445 & $13 \cdot 8$ & 445 & $15 \cdot 8$ & 0.972 \\
\hline$C(\mu \mathrm{m})$ & 323 & 9.5 & 326 & $10 \cdot 8$ & 0.797 \\
\hline$V+C(\mu \mathrm{m})$ & 767 & 20.4 & 772 & $23 \cdot 3$ & 0.892 \\
\hline$V / C$ & 1.40 & 0.041 & 1.38 & 0.047 & 0.827 \\
\hline Tela submucosa $(\mu \mathrm{m})$ & 50 & 1.9 & 61 & $2 \cdot 2$ & 0.002 \\
\hline Tunica muscularis $(\mu \mathrm{m})$ & 235 & $12 \cdot 4$ & 281 & $10 \cdot 9$ & 0.005 \\
\hline
\end{tabular}

$V$, villus height; $C$, crypt depth. 
Table 4. Gut digesta characteristics of fully weaned low birth weight (LBW) and normal birth weight (NBW) piglets (Mean values with their standard errors)

\begin{tabular}{|c|c|c|c|c|c|}
\hline \multirow[b]{2}{*}{ Variable } & \multicolumn{2}{|c|}{ LBW } & \multicolumn{2}{|c|}{ NBW } & \multirow[b]{2}{*}{$P$} \\
\hline & Mean & SE & Mean & SE & \\
\hline \multicolumn{6}{|l|}{ Stomach } \\
\hline $\mathrm{pH}$ & 3.44 & 0.164 & 3.67 & 0.187 & 0.353 \\
\hline Fresh matter $(\mathrm{g})$ & 225 & $17 \cdot 1$ & 258 & 19.5 & 0.205 \\
\hline Relative content (g/kg BW) & $24 \cdot 8$ & 1.64 & $22 \cdot 6$ & 1.88 & 0.366 \\
\hline \multicolumn{6}{|l|}{ Small intestine } \\
\hline \multicolumn{6}{|l|}{ Proximal } \\
\hline $\mathrm{pH}$ & 5.55 & 0.075 & $5 \cdot 50$ & 0.086 & 0.635 \\
\hline Fresh matter (g) & 57 & 6.4 & 67 & $7 \cdot 3$ & 0.273 \\
\hline Relative content (g/kg BW) & $6 \cdot 7$ & 0.81 & $6 \cdot 1$ & 0.93 & 0.608 \\
\hline \multicolumn{6}{|l|}{ Distal } \\
\hline $\mathrm{pH}$ & 6.96 & 0.069 & 6.92 & 0.079 & 0.705 \\
\hline Fresh matter $(\mathrm{g})$ & 70 & $6 \cdot 2$ & 89 & $7 \cdot 1$ & 0.062 \\
\hline Relative content (g/kg BW) & 7.9 & 0.63 & 7.7 & 0.72 & 0.784 \\
\hline Acetate $(\mu \mathrm{mol} / \mathrm{g})$ & 10 & $2 \cdot 7$ & 15 & $3 \cdot 1$ & 0.201 \\
\hline $\operatorname{SCFA}(\mu \mathrm{mol} / \mathrm{g})$ & 10 & $2 \cdot 7$ & 15 & $3 \cdot 1$ & 0.205 \\
\hline Lactate $(\mu \mathrm{mol} / \mathrm{g})$ & 26 & $6 \cdot 6$ & 27 & 7.5 & 0.962 \\
\hline \multicolumn{6}{|l|}{ Caecum } \\
\hline $\mathrm{pH}$ & 5.55 & 0.053 & 5.74 & 0.056 & 0.023 \\
\hline Fresh matter (g) & 57 & $6 \cdot 9$ & 71 & 7.9 & 0.173 \\
\hline Relative content (g/kg BW) & $6 \cdot 5$ & 0.74 & 6.4 & 0.84 & 0.912 \\
\hline Acetate $(\mu \mathrm{mol} / \mathrm{g})$ & 148 & $7 \cdot 4$ & 121 & $8 \cdot 4$ & 0.013 \\
\hline Propionate $(\mu \mathrm{mol} / \mathrm{g})$ & 57 & 3.9 & 47 & 3.4 & 0.040 \\
\hline Butyrate $(\mu \mathrm{mol} / \mathrm{g})$ & 19 & $1 \cdot 1$ & 19 & 1.3 & 0.786 \\
\hline SCFA $(\mu \mathrm{mol} / \mathrm{g})$ & 225 & $10 \cdot 5$ & 186 & $12 \cdot 0$ & 0.013 \\
\hline Acetate (\%) & 66 & $2 \cdot 4$ & 62 & 3.0 & 0.012 \\
\hline Propionate (\%) & 27 & 1.4 & 28 & 1.5 & 0.240 \\
\hline Butyrate (\%) & 8 & 0.5 & 9 & 0.6 & 0.423 \\
\hline Lactate $(\mu \mathrm{mol} / \mathrm{g})$ & 9 & 2.9 & 8 & $3 \cdot 2$ & 0.692 \\
\hline
\end{tabular}

BW, body weight.

either $C$ or $V$ or both, for the respective SI site (all $P<0 \cdot 001$ ). Overall, six histo-morphological variables were retained. The absolute and relative mass of digesta of each respective compartment were highly correlated (all $r>0.070 ; P<0 \cdot 001$ ). Distal SI pH was negatively related to distal SI lactate concentration $(r-0.58 ; P<0.001)$. All caecal SCFA concentrations were positively intercorrelated ( $r$ between 0.57 and 0.77 ; $P \leq 0.001$ ), while the relative amount of acetate (acetate/SCFA) in caecal contents had a negative relationship with propionate/SCFA and butyrate/SCFA $(r-0.79$ and -0.62 , respectively; $P<0.001)$. Only nine variables describing the digesta could be used in the final PCA. Short-circuit current responses to D-glucose, serotonin and theophylline were related, but only

Table 5. Plasma oxidative status of fully weaned low birth weight (LBW) and normal birth weight (NBW) piglets

(Mean values with their standard errors)

\begin{tabular}{|c|c|c|c|c|c|}
\hline \multirow[b]{2}{*}{ Variable } & \multicolumn{2}{|c|}{ LBW } & \multicolumn{2}{|c|}{ NBW } & \multirow[b]{2}{*}{$P$} \\
\hline & Mean & SE & Mean & SE & \\
\hline Malondialdehyde $(\mu \mathrm{M})$ & $7 \cdot 28$ & 0.247 & 7.53 & 0.283 & 0.497 \\
\hline $\operatorname{FRAP}\left(\mu \mathrm{M} \mathrm{Fe}^{2+}\right)$ & 107 & $2 \cdot 9$ & 114 & $2 \cdot 4$ & 0.049 \\
\hline GSH-Px* (U/ml) & 0.30 & 0.007 & 0.34 & 0.008 & $<0.001$ \\
\hline$\alpha$-Tocopherol $(\mu \mathrm{g} / \mathrm{ml})$ & 1.14 & 0.093 & 1.13 & $0 \cdot 106$ & 0.960 \\
\hline
\end{tabular}

FRAP, ferric reducing ability of plasma; GSH-Px, glutathione-peroxidase.

${ }^{*} \mathrm{U}$ : amount of GSH-Px required for oxidising $1 \mu \mathrm{mol}$ of $\mathrm{NADPH} / \mathrm{min}$ per $\mathrm{ml}$ plasma at $25^{\circ} \mathrm{C}$. the correlation between $\Delta \mathrm{IsC}_{\text {SEROTONIN }}$ and $\Delta \mathrm{ISC}_{\text {THEOPHYLLINE }}$ was higher than $0.55(r 0.79 ; P<0.001)$. The SI brush-border enzyme activities were significantly correlated within SI site, in particular within each enzyme category (peptidases $v$. carbohydrases). Only proximal aminopeptidase A and maltase and distal dipeptidylpeptidase IV and maltase were found to be representative for this group. Many variables describing the cytokine concentrations in the SI mucosa were correlated; however, only three of them were higher than 0.55 . These were proximal IL-6 $v$. proximal TNF- $\alpha(r 0.61 ; P<0 \cdot 001)$, proximal IL-6 $v$. proximal IL-10 $(r 0 \cdot 72 ; P<0 \cdot 001)$ and proximal IL-10 $v$. proximal TNF- $\alpha(r 0 \cdot 67 ; P<0 \cdot 001)$. No correlations were present between the plasma oxidative status parameters. Finally, thirtyfive variables were introduced for the PCA analysis (Table 6); and seven principal components explained $68.2 \%$ of the variance. The first principal component grouped the Ussing chamber results and in addition, some other variables such as proximal SI $V$, proximal SI TNF- $\alpha$ and plasma malondialdehyde had high loadings for this component. Negative loadings for some histo-morphological variables dominate in principal component 2, while this was the case for brush-border enzyme activities in principal component 3. In the latter component, distal SI acetate was associated in an opposite way as distal SI lactate; this was also seen in principal component 6. Principal component scores for principal components 4 and 5 were significantly different between LBW and NBW piglets; hence variables retained in these principal components are indicative 
Table 6. Description of the major principal components obtained by principal component analysis (PCA) of thirty-five variables characterising the gut function and oxidative status of fully weaned low birth weight (LBW) and normal birth weight (NBW) piglets $†$

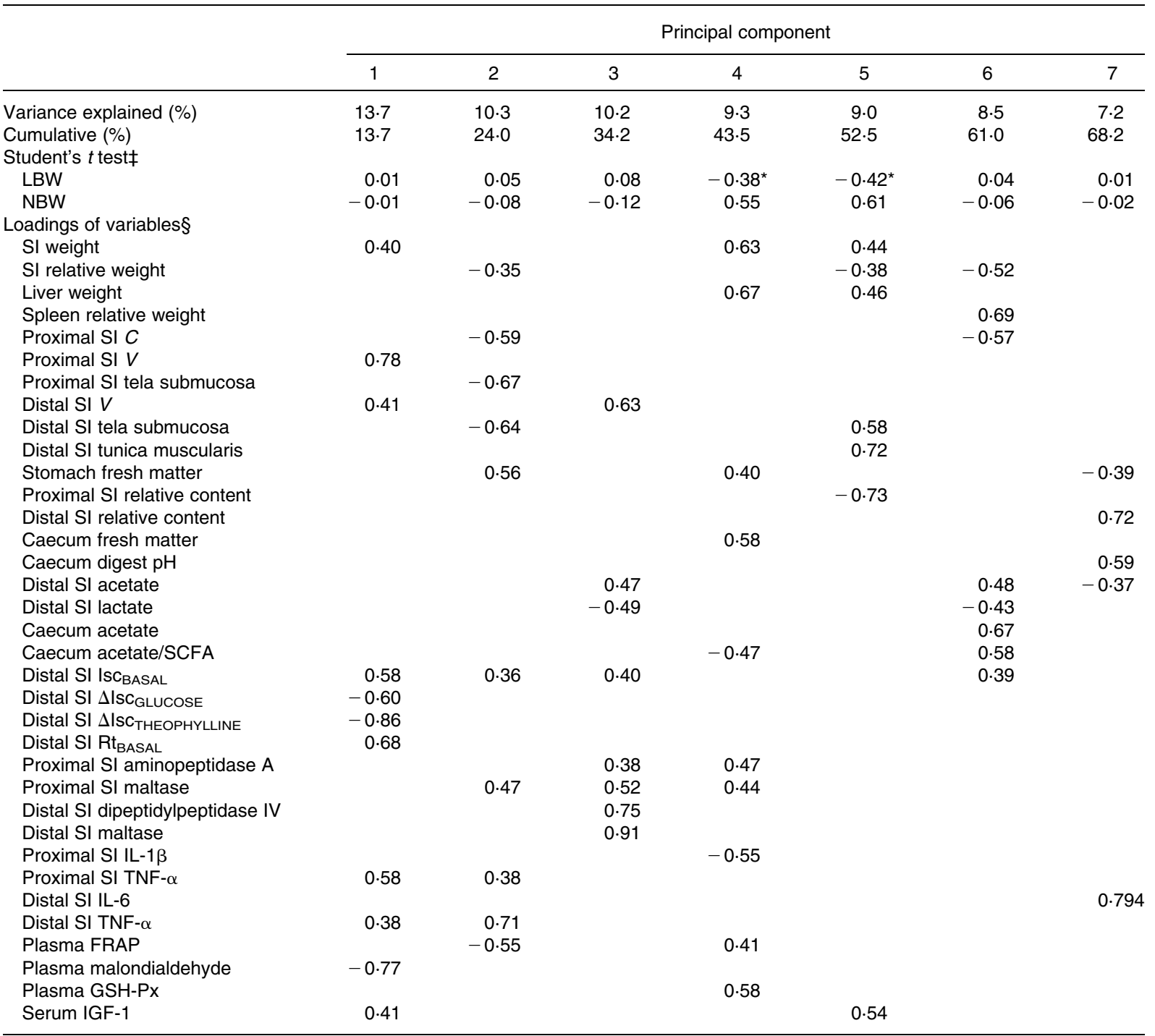

$\mathrm{SI}$, small intestine; $C$, crypt depth; $V$, villus height; Isc, short-circuit current; $\mathrm{Rt}_{\mathrm{BASAL}}$, basal tissue resistance; FRAP, ferric reducing ability of plasma; GSH-Px, glutathione-peroxidase; IGF-1, insulin-like growth factor-I.

*Values were significantly different $(P<0.01)$.

†Extraction method: PCA; rotation method: varimax with Kaiser normalisation.

$\ddagger$ Student's $t$ test on principal component scores of piglets.

$\S$ Only correlations with $|r|>0.35$ are indicated.

of physiological alterations in LBW piglets as compared to NBW piglets. Principal component 4 comprises weight of organs and digesta, along with variables related to brush-border enzyme activities in the proximal SI and variables presenting the plasma oxidative status (FRAP and GSH-Px). Both caecum acetate/SCFA and proximal SI IL-1 $\beta$ had a negative loading for component 4 . Similarly, principal component 5 deals with the absolute weight of organs (positive loadings) and relative weight of organs and digesta (negative loadings) and the distal SI histo-morphology (tela submucosa and tunica muscularis thickness). Importantly, this component was positively associated with serum IGF-1. Graphical representation of piglets according to their principal component scores for principal components 4 and 5 shows that piglets grouped fairly well within type of piglet (LBW $v$. NBW) and hence can be discriminated based on these principal components (Fig. 1). The remaining principal components contributed less to the variance explained and are more difficult to interpret.

\section{Discussion}

\section{Alterations in digestive function and plasma oxidative status of fully weaned low birth weight pigs}

Despite the fact that some interesting differences between the LBW and NBW piglets' digestive function were found, their number is far less than has been described for LBW pigs in 


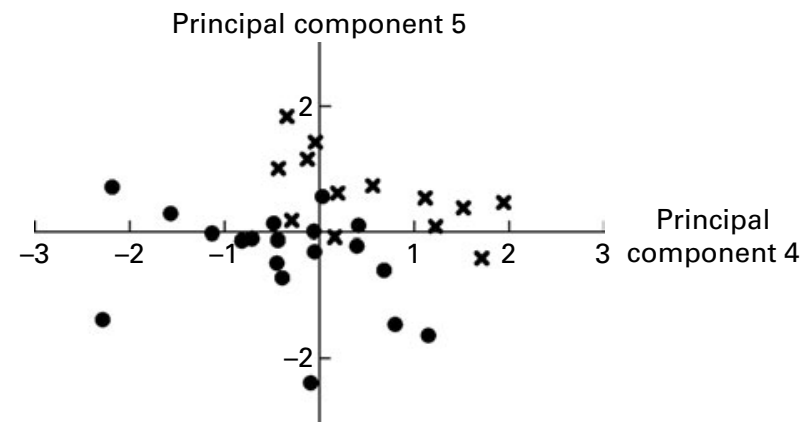

Fig. 1. Representation of low birth weight $(\bullet)$ and normal birth weight $(\mathbf{X})$ fully weaned piglets according to their principal component scores for principal components 4 and 5 from the principal component analysis. Animals can be discriminated visually according to type of piglet. See also Table 6 .

the neonatal stage. In fully weaned pigs, all digestive organs of LBW piglets were smaller with the exception of the spleen, SI weight-to-total length was lower, the distal SI tela submucosa and tunica muscularis were thinner, the secretory capacity differed from NBW pigs, caecal digesta were characterised by a reduced $\mathrm{pH}$ due to higher amounts of acetate and propionate, IGF-1R in SI tissues were less abundant, in particular in the proximal SI, and circulatory IGF-1 concentrations were markedly reduced. Most of these differences have been reported previously for the LBW or IUGR pig during the perinatal period and in LBW pigs not older than the weaning age ${ }^{(13,28,29)}$. These physiological alterations suggest that the normal developmental maturation of the GIT is retarded independent of the weaning process. The weaning event imposes severe dietary challenges to the animal, which results in an adaptation of the gut function. This typical adaptation does not seem to be affected by groups of pigs. Indeed, the GIT in pigs is profoundly affected by the weaning process; most documented are the changes in the $V$ and $C$ architecture and brush-border enzyme activities $^{(30-32)}$. Strangely, none of these parameters differed between LBW and NBW pigs. However, in the present study, pigs were sampled more than 2 weeks after weaning when most adaptations in response to weaning are completed ${ }^{(16)}$. Hence, we can conclude that in both groups of pigs (LBW $v$. NBW), the GIT adapted similarly upon weaning without persistent effects. Nevertheless, it has been repeatedly demonstrated that in contrast to the suckling period, LBW piglets have lower relative growth rates than their NBW littermates ${ }^{(6,33,34)}$. Probably, other than digestive shortcomings, factors such as sub-functional intermediate metabolism or hormonal imbalances might be involved in this reduction of growth potential.

Striking were the differences found for the caecum. A consistent lower $\mathrm{pH}$ of caecal contents in the LBW piglets was seen caused by higher acetate and propionate concentrations, which was accompanied by a higher relative acetate production. It is hard to explain these observations. Either more substrate was available to support fermentative activity or alternatively the bacterial composition could be changed as suggested by D'Inca et al. ${ }^{(13)}$. Here, we have no data to support any of these. Additional work is needed on various aspects of gastro-intestinal digestion to understand this better.
The Isc $\mathrm{BASAL}_{\mathrm{B}}$ in the distal SI was elevated in LBW piglets, which means that the SI mucosa of LBW pigs had a higher basal secretory response. As this response tends to decrease during the adaptive phase after weaning ${ }^{(30)}$, it confirms the delay in gut maturation.

To date, descriptions of changes of the oxidative status during the pigs' lifetime are rather fragmentary ${ }^{(35,36)}$. Here, in fully weaned LBW pigs, GSH-Px and FRAP were 13 and $7 \%$ lower, respectively, when compared with age-matched NBW pigs. This points to a lower reducing capacity of plasma upon reactive metabolites and oxidants when metabolically stressed. Nonetheless, one might disclaim the possible harmful consequences of a lower reducing capacity by stating that there is less need for reducing capacity in LBW pigs due to lower growth rates and lower feed intakes. Interestingly, Wang et $a l .{ }^{(37,38)}$ showed in a proteome analysis of gut mucosa that suckling IUGR pigs (0, 7 and $21 \mathrm{~d}$ of age) exhibited depressed levels of proteins that regulate oxidative defence and increased levels of proteins involved in the response to oxidative stress.

As already mentioned, less differences between LBW and NBW fully weaned pigs were observed compared to LBW neonates. During the suckling phase, $34.3 \%$ of the piglets, which were initially identified as LBW died (not determined in the NBW group). As a consequence, the population of fully weaned LBW pigs to be sampled here did not correspond to the LBW population at birth. Obviously, the weakest and less vital LBW piglets died, leaving less room to find physiological differences at a later age. In this respect, it is noteworthy to say that in modern pig breeding, efforts to support the survival and growth of LBW pigs are pertinent ${ }^{(8)}$ and hence research to understand the development of runting animals remains of paramount importance.

\section{Delayed gut maturation in low birth weight piglets}

As stated previously, the alterations in the digestive traits of fully weaned LBW piglets suggest a retardation in GIT maturation and do not seem to be related to the weaning transition as such. In addition to the changes that occur in response to the weaning process, some overlying developmental changes take place, independent of the weaning process ${ }^{(39)}$. The latter changes appear to be affected by birth weight. The PCA analysis (Table 6) comprehensively confirms this hypothesis. Principal components 4 and 5 bring together all variables that collectively discriminate LBW and NBW pigs (Fig. 1). Variables retained in the principal component 4 can be grouped into variables referring to weights of digestive organs and digesta. These variables have positive loadings and are thus reduced in LBW piglets; or they can be associated with the functioning of the proximal SI. The reduced proximal SI aminopeptidase A and, in particular, maltase activity (positive loadings) might support the assumption that in LBW pigs there is a delay in the normal developmental pattern of brush-border enzyme activities. Though not found to be different by the generalised linear model analysis, the negative loading $(-0.55)$ for proximal SI IL-1 $\beta$ suggests a higher pro-inflammatory response in LBW pigs. The negative association of GSH-Px and FRAP with LBW pigs (positive loadings and negative mean principal component 
scores) underlines the lower circulatory antioxidant capacity in this population of piglets. Analogously to principal component 4 , absolute organ weights had positive loadings in component 5. However, relative weight of SI and proximal SI relative content were negatively correlated with this principal component. The latter shows tentatively that LBW piglets have an elevated proximal SI fill when scaled to body weight. Knowing that absolute and relative weights of fresh matter in each compartment were highly and positively correlated, these suggest that LBW pigs have higher amounts of chyme in the proximal SI as compared to NBW pigs. This appears to be contradictory to the results from the generalised linear model analysis, which demonstrated that the absolute and relative weights of digesta within each compartment were not different between the two groups of pigs and numerically they were always lower for the LBW pigs. This contradiction might be related to the fact that no controlled feed intake protocol was used. Irrespective of that, the high and negative loading for the proximal SI relative content is appealing. Whittemore et al. ${ }^{(40)}$ demonstrated that relative gut fill (fresh matter content in the GIT/body weight) decreases with age. Then, the higher proximal SI relative fill would propose for a delayed gut development. Interestingly, the positive loadings for distal SI tela submucosa and tunica muscularis confirm the aforementioned findings. Again, it might indicate that gut maturation is retarded. Indeed, during maturation, the thickness of these layers and also of the mucosa increases to accommodate the processing of ingested feed $^{(41)}$. This study showed thinner intestinal layers in the case of LBW pigs.

Despite all this, the most remarkable variable that consistently differentiated LBW from NBW pigs was the serum IGF-1 level (positive loading in principal component 5). LBW pigs exhibited significantly lower IGF-1 concentrations in serum $(-20 \%)$. This is in line with data for LBW pigs preweaning $^{(42-44)}$ or at slaughter ${ }^{(3)}$; though in other studies systemic IGF-1 did not differ ${ }^{(33,44)}$. It is speculated that the growth-regulatory potential of IGF-1 might explain the developmental delay of GIT structure and function of LBW pigs in our experiment. Intravenous or subcutaneous administration of IGF-1 increased intestinal mucosal weight, protein and DNA content, $V$, and epithelial proliferation or reversed alterations upon challenge ${ }^{(45,46)}$. Additionally, the IGF-1R was less abundant in smooth muscle tissues (of the tunica muscularis and about submucosal arterioles) of LBW pigs compared with NBW littermates. The lower expression in the muscularis and submucosal arterioles corroborates with a thinner tela submucosa and tunica muscularis and could explain the lower SI weight:length ratio. Since IGF-1R in these tissues is already present at birth ${ }^{(47)}$, it can be suspected that the lower expression of IGF-1R in LBW pigs has its origin during impaired fetal growth. However, the present results are in contrast to recent data of Chen et $a l .{ }^{(48)}$ who did not find differences in IGF-1R expression in other tissues ( $\mathrm{m}$. longissimus dorsi, kidney and liver) of 21-d-old IUGR pigs. Thus, one might argue that despite the substantial and consistent decrease in serum IGF-1 and IGF-1R in SI tissues in our experiment, the effects on gut traits were rather limited. Perhaps, the temporal and sharp decrease in systemic IGF-1 and simultaneous increase in porcine somatotropin after weaning ${ }^{(49,50)}$ indicate that IGF-1 is less important for post-weaning development. This has been illustrated by Davis et al. ${ }^{(51)}$ and Dunshea ${ }^{(39)}$ who found no benefits of intravenous IGF-1 treatment in pigs at $26 \mathrm{~d}$ of age or post-weaning. Additionally, IGF-1 activity is lowered by the presence of IGF-binding proteins. If these proteins would be up-regulated, it could weaken the effect of IGF-1. Chen et $a l .{ }^{(48)}$ and Morise et $a l .{ }^{(33)}$ did not find differences in binding proteins in several tissues between IUGR pigs and NBW littermates pre-weaning. In the present experiment, IGF-binding proteins expression was not measured. Thus, there remains some lack in our understanding why LBW pigs fail to thrive, in particular in the post-weaning phase and what the involvement is of the IGF-1 system.

In conclusion, in fully weaned piglets, all digestive organs of LBW piglets were smaller with the exception of the spleen, SI weight-to-total length was lower, the distal SI tela submucosa and tunica muscularis were thinner, the secretory capacity differed from NBW pigs and caecal digesta were characterised by a reduced $\mathrm{pH}$ due to higher amounts of acetate and propionate. The few gut characteristics that were altered in fully weaned LBW piglets indicate a delay in the normal gut maturational process independent of the changes induced specifically by the weaning process. Evidence is brought about that lower circulatory IGF-1 concentrations and lower abundance of IGF-1R can account, at least partially, for these anomalies. Finally, the plasma antioxidant capacity was lower for the LBW pig, which opens up opportunities to optimise the provision of antioxidant nutrients. Probably, some of these alterations originate during fetal development. The results might aid to adjust feeding strategies for the LBW pig or to intervene in the maternal diet during gestation and lactation.

\section{Acknowledgements}

The authors wish to thank M. Bruggeman for allowing access to the pig breeding facilities. The authors are also grateful to F. Bruggeman, J. De Sutter, S. Coolsaet and S. Lescouhier for their dedicated and skilful technical support. The present work was partly supported by Onderzoeksfonds, College University Ghent. None of the authors has any conflict of interest. J. Mic., M. D. V. and C. V. G. planned and designed the study. J. Mic., M. D. V., J. Mis. and A. O conducted the experiment. J. Mic. and M. D. V. analysed the obtained data. J. Mic. and M. D. V. wrote the paper. J. Mic. had primary responsibility for the final content. All authors read, corrected and approved the final manuscript.

\section{References}

1. Herpin P, Le Dividich J, Hulin JC, et al. (1996) Effects of the level of asphyxia during delivery on viability at birth and early postnatal vitality of newborn pigs. J Anim Sci $\mathbf{7 4}$, 2067-2075.

2. Tuchscherer M, Puppe B, Tuchscherer A, et al. (2000) Early identification of neonates at risk: traits of newborn piglets with respect to survival. Theriogenology 54, 371-388. 
3. Gondret F, Lefaucheur L, Louveau L, et al. (2005) Influence of piglet birth weight on postnatal growth performance, tissue lipogenic capacity and muscle histological traits at market weight. Livest Prod Sci 93, 137-146.

4. Hoy S, Lutter C, Puppe B, et al. (1995) Correlations between vitality of newborn piglets, teat order, mortality, and live weight development up to weaning. Berl Munch Tierarztl 108, 224-228.

5. Milligan BN, Fraser D \& Kramer DL (2002) Within-litter birth weight variation in the domestic pig and its relation to preweaning survival, weight gain, and variation in weaning weights. Livest Prod Sci 76, 181-191.

6. Quiniou N, Dagorn J \& Gaudré D (2002) Variation of piglets' birth weight and consequences on subsequent performance. Livest Prod Sci 78, 63-70.

7. Foxcroft GR, Dixon WT, Novak S, et al. (2006) The biological basis for prenatal programming of postnatal performance in pigs. J Anim Sci 84, E105-E112.

8. Wu G, Bazer FW, Wallace JM, et al. (2006) Intrauterine growth retardation: implications for the animal sciences. J Anim Sci 84, 2316-2337.

9. Pere MC \& Etienne M (2000) Uterine blood flow in sows: effects of pregnancy stage and litter size. Reprod Nutr Develop 40, 369-382.

10. Wootton R, McFadyen IR \& Cooper JE (1997) Measurement of placental blood flow in the pig and its relation to placental and fetal weight. Neonatology 31, 333-339.

11. Su G, Lund MS \& Sorensen D (2007) Selection for litter size at day five to improve litter size at weaning and piglet survival rate. J Anim Sci 85, 1385-1392.

12. Le Dividich J, Martineau GP, Madec F, et al. (2003) Saving and rearing underprivileged and supernumerary piglets, and improving their health at weaning. In Weaning the Pig, Concepts and Consequences, pp. 361-383 [JR Pluske, J Le Dividich and MWA Verstegen, editors]. Wageningen, The Netherlands: Wageningen Academic Publishers.

13. D'Inca R, Kloareg M, Gras-Le Guen C, et al. (2010) Intrauterine growth restriction modifies the developmental pattern of intestinal structure, transcriptomic profile, and bacterial colonization in neonatal pigs. J Nutr 140, 925-931.

14. Flecknell PA, Wootton R, John M, et al. (1981) Pathological features of intrauterine growth-retardation in the piglet differential-effects on organ weights. Diagn Histopathol $\mathbf{4}$, 295-298.

15. Cooper JE (1975) The use of the pig as an animal model to study problems associated with low birth weight. Lab Anim 9, 329-336.

16. Montagne L, Boudry G, Favier C, et al. (2007) Main intestinal markers associated with the changes in gut architecture and function in piglets after weaning. Br J Nutr 97, 45-57.

17. Jamin A, D'Inca R, Le Floc'h N, et al. (2010) Fatal effects of a neonatal high-protein diet in low-birth-weight piglets used as a model of intrauterine growth restriction. Neonatology 97, 321-328.

18. Thornbury JC, Sibbons PD, Vanvelzen D, et al. (1993) Histological investigations into the relationship between low-birth-weight and spontaneous bowel damage in the neonatal piglet. Pediatr Pathol 13, 59-69.

19. Devillers N, Van Milgen J, Prunier A, et al. (2004) Estimation of colostrum intake in the neonatal pig. Anim Sci $\mathbf{7 8}$, 305-313

20. Van Nevel C, Decuypere JA, Dierick N, et al. (2003) The influence of Lentinus edodes (shiitake mushroom) preparations on bacteriological and morphological aspects of the small intestine in piglets. Arch Anim Nutr 57, 399-412.
21. Jensen MT, Cox RP \& Jensen BB (1995) Microbial production of skatole in the hind gut of pigs given different diets and its relation to skatole deposition in backfat. Anim Sci 61, 293-304.

22. Missotten JAM, Goris J, Michiels J, et al. (2009) Screening of isolated lactic acid bacteria as potential beneficial strains for fermented liquid pig feed production. Anim Feed Sci Technol 150, 122-138.

23. Michiels J, Missotten J, Dierick N, et al. (2010) Thymol and trans-cinnamaldehyde reduce active nutrient absorption and chloride secretion in the pig jejunal Ussing chamber model. Livest Sci 134, 27-29.

24. Benzie IFF \& Strain JJ (1996) The ferric reducing ability of plasma (FRAP) as a measure of "Antioxidant Power": the FRAP assay. Anal Biochem 239, 70-76.

25. Grotto D, Santa Maria LD, Boeira S, et al. (2007) Rapid quantification of malondialdehyde in plasma by high performance liquid chromatography-visible detection. J Pharm Biomed Anal 43, 619-624.

26. Hernández P, Zomeño L, Ariño B, et al. (2004) Antioxidant, lipolytic and proteolytic enzyme activities in pork meat from different genotypes. Meat Sci 66, 525-529.

27. Desai ID (1984) Vitamin E analysis methods for animal tissues. Methods Enzymol 105, 138-147.

28. Wang T, Huo YJ, Shi FX, et al. (2005) Effects of intrauterine growth retardation on development of the gastrointestinal tract in neonatal pigs. Biol Neonate 88, 66-72.

29. Xu RJ, Mellor DJ, Birtles MJ, et al. (1994) Impact of intrauterine growth-retardation on the gastrointestinal-tract and the pancreas in newborn pigs. J Pediatr Gastroenterol Nutr 18, 231-240.

30. Boudry G, Péron V, Le Huërou-Luron I, et al. (2004) Weaning induces both transient and long-lasting modifications of absorptive, secretory, and barrier properties of piglet intestine. J Nutr 134, 2256-2262.

31. Hedemann MS, Hojsgaard S \& Jensen BB (2003) Small intestinal morphology and activity of intestinal peptidases in piglets around weaning. J Anim Physiol Anim Nutr 87, 32-41.

32. Pluske JR. (2001) Morphological and functional changes in the small intestine of the newly-weaned pig. In Gut Environment in Pigs, pp. 1-27 [A Piva, KEB Knudsen and J-E Lindburg, editors]. Nottingham: Nottingham University Press.

33. Morise A, Seve B, Mace K, et al. (2009) Impact of intrauterine growth retardation and early protein intake on growth, adipose tissue, and the insulin-like growth factor system in piglets. Pediatr Res 65, 45-50.

34. Rezaei R, Knabe DA, Li X, et al. (2011) Enhanced efficiency of milk utilization for growth in surviving low-birth-weight piglets. J Anim Sci Biotechnol 2, 73-83.

35. Petrovic V, Novotny J, Hisira V, et al. (2009) The impact of suckling and post-weaning period on blood chemistry of piglets. Acta Vet BRNO 78, 365-371.

36. Sauerwein H, Schmitz S \& Hiss S (2005) The acute phase protein haptoglobin and its relation to oxidative status in piglets undergoing weaning-induced stress. Redox Rep 10, 295-302.

37. Wang J, Chen L, Li D, et al. (2008) Intrauterine growth restriction affects the proteomes of the small intestine, liver, and skeletal muscle in newborn pigs. $J$ Nutr 138, $60-66$

38. Wang XQ, Wu WZ, Lin G, et al. (2010) Temporal proteomic analysis reveals continuous impairment of intestinal development in neonatal piglets with intrauterine growth restriction. J Proteome Res 9, 924-935.

39. Dunshea FR (2003) Metabolic and endocrine changes around weaning. In Weaning the Pig, Concepts and 
Consequences, pp. 61-80 [JR Pluske, J Le Dividich and MWA Verstegen, editors]. Wageningen: Wageningen Academic Publishers.

40. Whittemore EC, Emmans GC \& Kyriazakis I (2003) The relationship between live weight and the intake of bulky foods in pigs. Anim Sci 76, 89-100.

41. Adeola O \& King DE (2006) Developmental changes in morphometry of the small intestine and jejunal sucrase activity during the first nine weeks of postnatal growth in pigs. J Anim Sci 84, 112-118.

42. Dauncey MJ, Burton KA \& Tivey DR (1994) Nutritional modulation of insulin-like growth factor-I expression in early postnatal piglets. Pediatr Res 36, 77-83.

43. Ritacco G, Radecki SV \& Schoknecht PA (1997) Compensatory growth in runt pigs is not mediated by insulin-like growth factor I. J Anim Sci 75, 1237-1243.

44. Schoknecht PA, Ebner S \& Skottner A (1997) Exogenous insulin-like growth factor-I increases weight gain in intrauterine growth-retarded neonatal pigs. Pediatr Res $\mathbf{4 2}$, 201-207.

45. Peterson CA, Ney DM, Hinton PS, et al. (1996) Beneficial effects of insulin-like growth factor I on epithelial structure and function in parenterally fed rat jejunum. Gastroenterology 111, 1501-1508.

46. Read LC, Tomas FM, Howarth GS, et al. (1992) Insulin-like growth factor-I and its N-terminal modified analogs induce marked gut growth in dexamethasone-treated rats. $J$ Endocrinol 133, 421-431.

47. Van Ginneken C, Haver EV, Oste M, et al. (2007) The presence of EGF- and IGF-1-receptors in the small intestine of fetal, neonatal and weaned piglets. Livest Sci 108, 57-60.

48. Chen R, Yin Y, Pan J, et al. (2011) Expression profiling of IGFs and IGF receptors in piglets with intrauterine growth restriction. Livest Sci 136, 72-75.

49. Carroll JA, Veum TL \& Matteri RL (1998) Endocrine responses to weaning and changes in post-weaning diet in the young pig. Domest Anim Endocrinol 15, 183-194.

50. White ME, Ramsay TG, Osborne JM, et al. (1991) Effect of weaning at different ages on serum insulin-like growthfactor I (IGF-I), IGF binding proteins and serum in vitro mitogenic activity in swine. J Anim Sci 69, 134-145.

51. Davis TA, Fiorotto ML, Burrin DG, et al. (2002) Acute IGF-I infusion stimulates protein synthesis in skeletal muscle and other tissues of neonatal pigs. Am J Physiol Endocrinol Metabol 283, E638-E647. 